\title{
Assessment of the macroinvertebrate community of the Vjosa river through non-destructive DNA metabarcoding of preservative ethanol
}

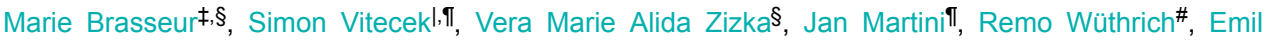

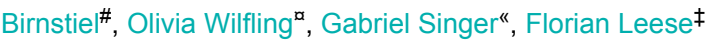 \\ ‡ University of Duisburg-Essen, Essen, Germany \\ § Zoological Research Museum Alexander Koenig, Bonn, Germany \\ | Senckenberg Research Institute and Natural History Museum, Frankfurt am Main, Germany \\ I 2 WasserCluster Lunz - Biol. Station GmbH, Lunz, Austria \\ \# gutwasser $\mathrm{GmbH}$, Zurich, Switzerland \\ - University of Natural Resources and Life Sciences, Vienna, Austria \\ "Leibniz-Institute of Freshwater Ecology and Inland Fisheries (IGB), Berlin, Germany
}

Corresponding author: Marie Brasseur (m.brasseur21@gmail.com)

Received: 23 Feb 2021| Published: 04 Mar 2021

Citation: Brasseur M, Vitecek S, Zizka VMA, Martini J, Wüthrich R, Birnstiel E, Wilfling O, Singer G, Leese F

(2021) Assessment of the macroinvertebrate community of the Vjosa river through non-destructive DNA metabarcoding of preservative ethanol. ARPHA Conference Abstracts 4: e64865.

https://doi.org/10.3897/aca.4.e64865

\begin{abstract}
Streams and rivers represent hotspots of biodiversity in their natural state. This biodiversity is declining worldwide due to pollution, exploitation and hydromorphological degradation of these systems. One of the last big, natural rivers in Europe is the Vjosa in the Balkan region. The catchment is characterized by natural flow dynamics, resulting in high habitat diversity and turnover, and hosts several sensitive and endemic species (e.g. Isoperla vjosae). DNA metabarcoding represents a promising approach to characterize this biodiversity but methodological drawbacks such as primer bias or incomplete reference databases limit the application, particularly in taxonomically underexplored regions.

Here, we assessed stream biodiversity with a focus on macrozoobenthic (MZB) taxa via a non-destructive, voucher-preserving DNA metabarcoding protocol. In this approach, ethanol used for preservation of multi-habitat samples in the field was used as DNA
\end{abstract}


template, allowing to retain the integrity of the original sample and further comparison of molecular and morphological taxa lists. Samples were taken in spring and autumn 2018 at 48 sites allocated over the Vjosa catchment. The preservative ethanol was filtered through 0.43 um nitrocellulose membranes from which DNA was extracted. Subsequently, a $421 \mathrm{bp}$ fragment of the COI gene was amplified with the primer pair BF2/BR2 and Illumina sequenced. After filtering for sequences with similarity to reference entries $>85 \%, 4,123$ OTUs were obtained, of which 921 were identified as MZB taxa. Dipterans and ephemeropterans were most abundant, followed by plecopterans. Some taxa (e.g. molluscs) were not identified due to a known primer bias and $>7,000$ OTUs could not be assigned above $85 \%$ similarity. Using the here presented voucher-preserving approach allowed us to identify the pitfalls of DNA metabarcoding as tool for biodiversity assessment in taxonomically unexplored regions such as the Vjosa catchment. However, the comparison of specimen abundance data and molecular data showed the power of nondestructive fixative metabarcoding for detecting MZB communities with highly increased taxonomic resolution.

\section{Keywords}

free DNA, fixative, voucher-preserving, biodiversity assessment

\section{Presenting author}

Marie Brasseur

\section{Presented at}

1st DNAQUA International Conference (March 9-11, 2021) 